\title{
Willingness to pay for morbidity and mortality risk reductions during an epidemic. Theory and preliminary evidence from COVID-19
}

\author{
Luciana Echazu ${ }^{1}$. Diego C. Nocetti ${ }^{2}$
}

Received: 15 May 2020 / Accepted: 31 July 2020 / Published online: 13 August 2020

(c) International Association for the Study of Insurance Economics 2020

\begin{abstract}
The COVID-19 pandemic and the strong social distancing measures adopted by governments around the world provide an ideal scenario to evaluate the trade-off between lives saved and morbidity avoided on the one hand and reduced economic resources on the other. We adapt the standard model of willingness to pay (WTP) for mortality/ morbidity risk reductions by incorporating a number of aspects that are highly relevant during an epidemic; namely, health-care capacity constraints, dynamic aspects of prevention (i.e., interventions aimed at flattening the epidemic curve), and distributional issues due to high heterogeneity in the underlying risks. The calibration of the model generates a WTP of the order of $24 \%$ of GDP. We conclude that the benefits in terms of lives saved and morbidity avoided can well justify the enormous economic costs generated by social distancing interventions. There is, however, significant that heterogeneity in WTP estimates depending on the degree of vulnerability to infection risk (e.g., by age), implying a large redistribution of income and well-being.
\end{abstract}

Keywords Willingness to pay · Value of statistical injury · COVID-19 pandemic . Health-care capacity constraints

\section{Introduction}

As a result of the ongoing coronavirus (COVID-19) pandemic, individuals and governments around the world are undertaking strong actions to prevent the rapid spread of the disease and potentially millions of deaths, including school and

Diego C. Nocetti

dnocetti@clarkson.edu

Luciana Echazu

luciana.echazu@unh.edu

1 Peter Paul College of Business and Economics, University of New Hampshire, Durham, NH, USA

2 David D. Reh School of Business, Clarkson University, Potsdam, NY, USA

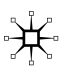


universities closures, stay-at-home mandates, and even lock downs of entire cities. These actions generate a steep cost in terms of reduced economic activity, millions of lost jobs, worse educational outcomes for children, psychological stress, reduced health-care resources for other medical conditions, and many others. The evaluation of the costs and benefits of preventing fatalities and/or non-fatal injuries has a long history in economics [See Viscusi (2014) for a recent treatment]. To capture the trade-off between economic resources and human lives saved, economists typically calculate the value of statistical life (VSL): The amount that individuals are willing to pay for an intervention that marginally reduces mortality risk, per unit of risk. Similarly, the value of statistical injury (VSI) represents the amount that individuals are willing to bear to reduce the risk of an injury or a disease, per unit of risk. The COVID-19 pandemic and the measures adopted provide an ideal scenario to apply these methods and to evaluate the balance between the revealed value of the lives saved and morbidity avoided on the one hand and the costs in terms of reduced economic resources on the other (and possibly opportunity costs and non-pecuniary costs as well).

We adapt the standard economic model of willingness to pay (WTP) for morbidity and fatality risk reductions by incorporating a number of issues that are particularly relevant during epidemics [see Anderson et al. (2020) for a discussion of these issues from an epidemiological perspective]; namely, health-care capacity constraints, dynamic aspects of prevention (i.e., interventions aimed at flattening the epidemic curve to avoid overwhelming the health-care system and to allow time for vaccination discovery and manufacture of antiviral drug therapies), and distributional issues due to high heterogeneity in the underlying risks.

Our theoretical exercise shows that static and dynamics aspects of prevention interact in interesting ways to determine the WTP for a public intervention that reduces the risk of virus contagion across the population (e.g., social distancing/isolation measures). For example, when vaccine discovery becomes more likely (i.e., a shorter expected time until vaccine discovery), there is a stronger incentive to reduce the current spread of the virus but at the same time there is a reduction in the risk of being infected over the course of the pandemic, so the effect on the WTP is ambiguous. Conversely, while reducing the probability of infection typically becomes more valuable the higher the infection rate is, individuals may be willing to sacrifice less economic resources for a given intervention if the risk of infection over the course of the pandemic remains relatively unchanged (e.g., when the infection risk remains high after the intervention and vaccine discovery is unlikely to happen in the near term). To put it differently, we should observe more support for social distancing measures when the probability of infection is higher (e.g., New York City versus Houston in March 2020), insofar as those interventions make a sufficiently large difference in terms of averting morbidity and mortality over the entire course of the pandemic.

As expected, a larger size of the vulnerable population or more limited healthcare resources increase the WTP, and both aspects reinforce each other as those more vulnerable are more likely to suffer from health-care capacity shortages. The effect of limited health-care capacity is particularly strong when vaccine discovery 
is unlikely in the near future and when people are more patient since in this case future capacity shortages become more relevant in the present.

We use currently available epidemiological data to calibrate the model. For an intervention that reduces the baseline probability of infection by $90 \%$, we obtain a maximum willingness to pay of about $\$ 15,500$ per person in the United States $(\$ 5.0$ trillion, or about $24 \%$ of 2019 GDP), with a range of $\$ 8000$ to $\$ 28,000$ per person under alternative parametrizations. The calibration generates a "social" Value of Statistical Injury (aggregate WTP per expected morbidity avoided) of about $\$ 34,000$, which is consistent, although in the lower range of VSI estimates typically found in the literature [e.g., Viscusi and Aldy (2003)].

The possibility of vaccine discovery and health-care capacity constraints both play an important role in the baseline calibration, each contributing a significant portion of WTP. In addition, we present suggestive evidence that, due to pronounced vulnerability differences, there are large differences in WTP. In the baseline calibration, the high vulnerability group has a WTP of more than $\$ 40,000$ while the low vulnerability group's WTP is only about $\$ 11,000$.

The paper proceeds as follows. In the next section, we present the model and evaluate the comparative statics of then model's parameters. In Sect. 3, we present a calibration of the model while Sect. 4 discusses the limitations of the analysis and concludes. An Appendix presents the derivations of the main equations presented in the text.

\section{Theoretical issues}

The standard model behind willingness to pay for mortality risk reductions is well known [see e.g., Anderson and Treich (2011) for a review of theoretical insights]. Given a baseline probability of dying $p$ and a utility over wealth $u(W)$ if alive and $d(W)$ if dead, a decision-maker's expected utility is $V\{p\}=(1-p) u(W)+p d(W)$ and her willingness to pay $C(\sigma)$ to decrease the risk by a proportion $\sigma$ is given by

$$
(1-p) u(W)+p d(W)=(1-p(1-\sigma)) u(W-C(\sigma))+p(1-\sigma) d(W-C(\sigma)) .
$$

If, as we assume below, the decision-maker is risk neutral, $u(W)=W$, and $d(W)=0$, we have

$$
C(\sigma)=\frac{\sigma p W}{(1-p(1-\sigma))} .
$$

A first-order approximation around $\sigma=0$ gives

$$
C(\sigma) \approx C(0)+C^{\prime}(0) \sigma=\frac{W}{(1-p)} \sigma p
$$

The term $\frac{W}{(1-p)}$, and its equivalent expression as the marginal rate of substitution between wealth and crude mortality risk $\frac{\mathrm{d} W}{\mathrm{~d} p} \mathrm{~d} V=0$, is known as the Value of a Statistical Life (VSL). 
Pandemics such as COVID-19 are characterized by a number of issues that are not well-captured by Eq. (1). First, both mortality and morbidity risks play a significant role. Second, given the number of people potentially affected, any intervention has to take into account capacity constraints of the health-care system. Third, the underlying risks and the effects of interventions may be highly heterogeneous across the population (for instance, in the case of COVID-19, mortality and morbidity are highly correlated with age and pre-existing conditions), bringing distributional issues into consideration. Finally, decision makers (individuals and/or the government) have limited direct control over mortality risk of those infected; instead, early interventions typically aim at limiting social contacts to contain and/or mitigate the rapid spread of the virus (e.g., due to capacity constraints and the possibility of vaccine discovery). This introduces dynamic aspects which are not present in Eq. (1).

In the next two subsections we evaluate each of these aspects, first one at a time for clarity and then all together.

\subsection{Static analysis}

We are interested in evaluating the willingness to pay to reduce mortality and morbidity risks during an epidemic, from the time in which preventive actions are considered onwards. We start by considering a static framework as above.

\subsubsection{Basic setup}

Consider a population of $M$ individuals, all of which are susceptible to a virus infection. The infection risk is $p_{\mathrm{I}}$. Of those infected, a proportion $p_{\mathrm{S}}$ become severely ill and require hospitalization (provided that hospital capacity is available). Among those hospitalized, a proportion $p_{\mathrm{D}}$ die and the rest recover. The crude mortality risk therefore is $p=p_{\mathrm{I}} p_{\mathrm{S}} p_{\mathrm{D}}$. Utility equals $W$ if not severely sick, $m W$ if severely sick, where $m<1$ represents a utility cost of morbidity and/or a fraction of wealth lost due to sickness, and 0 if dead. Given the assumed probabilities, and without any other consideration (e.g., capacity constraints, heterogeneity, dynamics), expected utility for any given individual equals

$$
V^{b}\left\{p_{\mathrm{I}}\right\}=\left(1-p_{\mathrm{I}} p_{\mathrm{S}}\right) W+p_{\mathrm{I}} p_{\mathrm{S}}\left(1-p_{\mathrm{D}}\right) m W .
$$

Consider an intervention that reduces social contact and, as such, decreases infection risk by a proportion $\sigma$. The willingness to pay for this intervention is

$$
C^{b}(\sigma)=\frac{V^{b}\left\{p_{\mathrm{I}}(1-\sigma)\right\}-V^{b}\left\{p_{\mathrm{I}}\right\}}{\left(1-(1-\sigma) p_{\mathrm{I}} p_{\mathrm{S}}\right)+(1-\sigma) p_{\mathrm{I}} p_{\mathrm{S}}\left(1-p_{\mathrm{D}}\right) m}
$$

or equivalently,

$$
C^{b}(\sigma)=\frac{\sigma p_{\mathrm{I}} p_{\mathrm{S}}\left(1-\left(1-p_{\mathrm{D}}\right) m\right)}{\left(1-(1-\sigma) p_{\mathrm{I}} p_{\mathrm{S}}\right)+(1-\sigma) p_{\mathrm{I}} p_{\mathrm{S}}\left(1-p_{\mathrm{D}}\right) m} W
$$


$C^{b}(\sigma)$ increases with the baseline probability of infection $p_{\mathrm{I}}$, the probability of a severe case $p_{\mathrm{S}}$, and the probability that a severely sick individual dies $p_{\mathrm{D}}$. A larger morbidity discount, or equivalently a higher cost of hospitalization, (i.e., a lower $m$ ) also increases WTP.

\subsubsection{Capacity constraints}

Let $K$ denote the maximum capacity available for coronavirus patients. Suppose that if maximum capacity is overwhelmed, those that are severely ill but are not hospitalized die at a rate $p_{\mathrm{DK}}>p_{\mathrm{D}}$. Assuming random selection of those that are hospitalized if the constraint binds, the probability of hospitalization is $p_{h}=\min \left(1, \frac{K}{p_{\mathrm{S}} p_{\mathrm{I}} M}\right)$. Define the capacity gap as $G=p_{\mathrm{S}} p_{\mathrm{I}} M-K{ }^{1}$ Utility can be written as

$$
V^{K}\left\{p_{\mathrm{I}}\right\}=V^{b}\left\{p_{\mathrm{I}}\right\}-m\left(p_{\mathrm{DK}}-p_{\mathrm{D}}\right) W \max (G, 0) / M
$$

Capacity gaps, and the resulting increase in infection mortality rate, reduce utility and the size of the decrease is proportional to the capacity gap per capita, $\max (G, 0) / M$. The WTP for a proportional reduction in infection risk in this case is

$$
C^{K}(\sigma)=\frac{\sigma p_{\mathrm{I}} p_{\mathrm{S}}\left(1-\left(1-p_{\mathrm{D}}\right) m\right)+m\left(p_{\mathrm{DK}}-p_{\mathrm{D}}\right)\left(\max (G, 0) / M-\max \left(G^{\prime}, 0\right) / M\right)}{\left(1-(1-\sigma) p_{\mathrm{I}} p_{\mathrm{S}}\right)+(1-\sigma) p_{\mathrm{I}} p_{\mathrm{S}}\left(1-p_{\mathrm{D}}\right) m-m\left(p_{D, K}-p_{\mathrm{D}}\right) \max \left(G^{\prime}, 0\right) / M} W,
$$

where $G^{\prime}=p_{\mathrm{S}} p_{\mathrm{I}}(1-\sigma) M-K$ is the capacity gap post intervention. Since $\max (G, 0) \geq \max \left(G^{\prime}, 0\right) \geq 0$, it follows that capacity gaps, and in particular lower capacity available $K$, increase the WTP. A larger difference in mortality risk from insufficient capacity, $\left(p_{\mathrm{DK}}-p_{\mathrm{D}}\right)$, also increases WTP. For example, given a reduction in infection risk that is just enough to eliminate an existing capacity gap, i.e., $G^{\prime}=0$ and $\sigma=G / p_{\mathrm{I}} p_{\mathrm{S}} M$, we have

$$
C^{K}(\sigma)=C^{b}(\sigma)+\frac{m\left(p_{\mathrm{DK}}-p_{\mathrm{D}}\right)(G / M)}{1-(K / M)\left[1-\left(1-p_{\mathrm{D}}\right) m\right]} W .
$$

\subsubsection{Heterogeneous vulnerabilities}

Consider three types of individuals: immune, low risk $(l)$, and high risk $(h)$. There are $M-N$ immune individuals with utility $W$. Among the $N$ susceptible individuals,

\footnotetext{
1 To avoid additional notation, we assume that $m$ is the same for individuals that are not hospitalized. The idea is that, while those individuals may have a larger utility cost, they will also have a lower monetary cost.
} 
those of type $h$ are in proportion $\alpha$ and have an additional probability of severe illness $\omega_{\mathrm{S}}$ and an additional probability of mortality of severe cases $\omega_{\mathrm{D}}$ than type $l$ .$^{2}$ The expected utility for type $l$ individuals is, as before, $V^{b}\left\{p_{\mathrm{I}}\right\}$ and we denote $V^{h}\left\{p_{\mathrm{I}}\right\}$ the expected utility for type $h$ individuals. Given a utilitarian social welfare function $V^{S}\left\{p_{\mathrm{I}}\right\}^{3}$, we have

$$
V^{S}\left\{p_{\mathrm{I}}\right\}=(M-N) W+N\left[(1-\alpha) V^{b}\left\{p_{\mathrm{I}}\right\}+\alpha V^{h}\left\{p_{\mathrm{I}}\right\}\right] .
$$

Let us suppose that everybody pays the same amount $C^{S}(\sigma)$ to reduce infection risk by a proportion $\sigma .{ }^{4}$ The amount $C^{S}(\sigma)$ that leaves social welfare unchanged can be written as a weighted average of each group's WTP,

$$
C^{S}(\sigma)=q_{l} C^{b}(\sigma)+q_{h} C^{h}(\sigma)+\left(1-q_{l}-q_{h}\right) 0,
$$

$$
\begin{gathered}
\text { where } \quad q_{h}=\frac{\alpha N V^{h}\left\{p_{\mathrm{I}}(1-\sigma)\right\}}{(M-N) W+N\left[(1-\alpha) V^{b}\left\{p_{\mathrm{I}}(1-\sigma)\right\}+\alpha V^{h}\left\{p_{\mathrm{I}}(1-\sigma)\right\}\right]} \\
q_{l}=\frac{(1-\alpha) N V^{b}\left\{p_{\mathrm{I}}(1-\sigma)\right\}}{(M-N) W+N\left[(1-\alpha) V^{b}\left\{p_{\mathrm{I}}(1-\sigma)\right\}+\alpha V^{h}\left\{p_{\mathrm{I}}(1-\sigma)\right\}\right]} .
\end{gathered}
$$

Not surprisingly, since $W>V^{b}>V^{h}, C^{S}(\sigma)$ increases as the number of susceptible individuals $N$ increases (e.g., an earlier intervention) and also as the share of more vulnerable individuals $\alpha$ increases (e.g., countries with older population). ${ }^{5}$

\subsubsection{A simple dynamic setting}

In the static framework above, we assumed that a fraction of the susceptible population is infected but we did not consider what happens with the remaining susceptible individuals. In reality, interventions reducing social contact slow down, but do not necessarily reduce or stop, the spread of the disease. Here we consider two reasons why flattening the epidemic curve may be useful. First, it allows time for vaccination discovery and manufacture of antiviral drug therapies. Second, reducing the initial spread of the disease may reduce or eliminate health-care capacity gaps.

To capture these issues in a simple way, suppose that in any given period, $t=1,2, .$. , susceptible individuals become infected at a constant rate $p_{\mathrm{I}}$ and surviving individuals become immune to the virus. The time from infection to recovery or death is no more than one period, although presumably some individuals spread the virus from one period to the next. This means that period $t$ starts with $\left(1-p_{\mathrm{I}}\right)^{t-1} N$ susceptible individuals and has $p_{\mathrm{I}}\left(1-p_{\mathrm{I}}\right)^{t-1} N$ new cases (given that a vaccine has

\footnotetext{
${ }^{2}$ In our complete model, we will denote $\omega_{\mathrm{DK}}$ the additional probability of mortality given no hospitalization (given capacity constraints).

${ }^{3}$ See Adler et al. (2014) for an analysis of how the value of risk reduction is evaluated under social preferences other than utilitarian.

${ }^{4}$ A uniform payment $C^{S}(\sigma)$ may be justified if the social planner does not have information about the individual types and/or if there are large (unmodeled) costs of adopting differential policies-Treich and Yang (2019) provide a thorough discussion of the financing of public safety projects in the context of imperfect tax systems. We recognize, however, that our assumption does not capture the fact that people may face different costs from uniform public prevention interventions (e.g., Restrictions on travel and non-essential businesses may have a more limited impact on the elderly).

${ }^{5}$ Notice that, in our setting with a utilitarian social welfare function, greater heterogeneity in $p_{\mathrm{S}}$, in the sense of groups that generate a mean preserving spread over $p_{\mathrm{S}}$, would not change the social WTP.
} 
not been discovered). Periods can be interpreted as epidemic phases, each lasting two or three months after the intervention is considered at the beginning of $t=1$. This is important because the assumption that $p_{\mathrm{I}}$ is constant implies that the number of new cases at the end of the initial period, $p_{\mathrm{I}} N$, represents either the peak of the epidemic curve or the declining stage of the curve. In the calibration below, we assume that infection risk $p_{\mathrm{I}}$ depends on the number of infected individuals relative to the size of the population before the public intervention is considered (e.g., during COVID-19, many people were infected in New York City before strong measures were put in place, implying a relatively high $p_{\mathrm{I}}$ and a significant portion of the population becoming immune at $t=0$ ).

We assume that there is a constant probability $\beta$ that a vaccine is discovered in period $t$; i.e., the probability that a vaccine has not been discovered after $t$ periods equals $(1-\beta)^{t} .^{6}$ This implies that the expected number of deaths over the course of the epidemic equals $N_{\mathrm{D}}=\frac{p_{\mathrm{I}} p_{\mathrm{S}} p_{\mathrm{D}}}{1-(1-\beta)\left(1-p_{\mathrm{I}}\right)} N$ and the expected number of people infected over the course of the pandemic equals $N_{\mathrm{I}}=\frac{p_{\mathrm{I}}}{1-(1-\beta)\left(1-p_{\mathrm{I}}\right)} N$. Finally, suppose that the period discount factor for all individuals is $(1+r)^{-1}$, that period income equals $w$, and that the period morbidity discount $m$ applies to all infected individuals equally.

\subsubsection{Basic setup}

Going back to the most basic setting, but incorporating the possibility of vaccine discovery, lifetime expected utility can be written in recursive form as

$$
V_{t}\left\{p_{\mathrm{I}}\right\}=\left(1-p_{\mathrm{I}}\right)\left[w+\beta \frac{w}{r}+\frac{1-\beta}{(1+r)} V_{t+1}\left\{p_{\mathrm{I}}\right\}\right]+p_{\mathrm{I}}\left(1-p_{\mathrm{S}} p_{\mathrm{D}}\right) w\left(m+\frac{1}{r}\right)
$$

An individual that has just avoided infection (probability $\left(1-p_{\mathrm{I}}\right)$ ) receives income $w$ and expects to receive $w$ thereafter if a vaccine is discovered (expected present value $\beta \frac{w}{r}$ ) and $\frac{1}{(1+r)} V_{t+1}$ if it is not. An individual that is infected, whether severely or not, and does not die (probability $p_{\mathrm{I}}\left(1-p_{\mathrm{D}} p_{\mathrm{S}}\right)$ ) receives utility $w\left(m+\frac{1}{r}\right)$.

Setting $V_{t}\left\{p_{\mathrm{I}}\right\}=V_{t+1}\left\{p_{\mathrm{I}}\right\} \equiv V^{d}\left\{p_{\mathrm{I}}\right\}$ in Eq. (8) we obtain

$$
V^{d}\left\{p_{\mathrm{I}}\right\}=\frac{(1+r)}{r+p_{\mathrm{I}}+\beta\left(1-p_{\mathrm{I}}\right)} w\left[\left(1-p_{\mathrm{I}}\right)\left(1+\beta \frac{1}{r}\right)+p_{\mathrm{I}}\left(1-p_{\mathrm{S}} p_{\mathrm{D}}\right)\left(m+\frac{1}{r}\right)\right]
$$

We evaluate the WTP out of first period income, now denoted $c^{d}(\sigma)$, to reduce $p_{\mathrm{I}}$ (in all periods) by a proportion $\sigma$. The interpretation of the intervention parameter $\sigma$ is not necessarily that the same social distancing measures remain forever; rather, it seems likely that maintaining a lower, constant infection rate requires strong early actions followed by an easing of the policy measures over time. We allow $c^{d}(\sigma)$ to

\footnotetext{
${ }^{6}$ The parameter $\beta$ may also capture the progressive discovery of efficient antiviral treatments-reducing expected death and morbidity costs for susceptible individuals.
} 


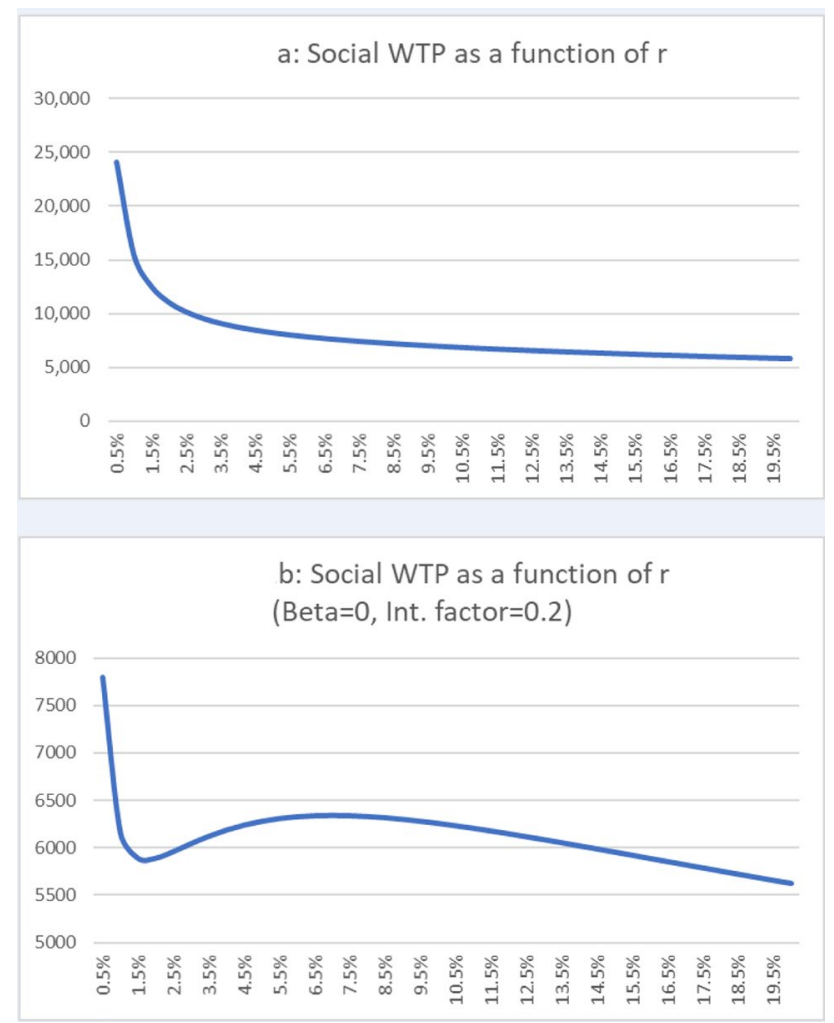

Fig. 1 a Social WTP as a function of $r$. b Social WTP as a function of $r$ (beta $=0$; int. factor $=0.2$ )

be larger than $w$, so borrowing is allowed, but the assumption that WTP is valued in current dollars means that the expected net present value of such borrowing must be zero (see e.g., Shepard and Zechhauser 1984). The maximum WTP for the susceptible group is

$$
V^{d}\left\{p_{\mathrm{I}}\right\}=V^{d}\left\{p_{\mathrm{I}}(1-\sigma)\right\}-\left[\left(1-p_{\mathrm{I}}(1-\sigma)\right)+p_{\mathrm{I}}(1-\sigma)\left(1-p_{\mathrm{S}} p_{\mathrm{D}}\right) m\right] c^{d}(\sigma),
$$

or equivalently,

$$
c^{d}(\sigma)=\frac{V^{d}\left\{p_{\mathrm{I}}(1-\sigma)\right\}-V^{d}\left\{p_{\mathrm{I}}\right\}}{\left(1-p_{\mathrm{I}}(1-\sigma)\right)+p_{\mathrm{I}}(1-\sigma)\left(1-p_{\mathrm{S}} p_{\mathrm{D}}\right) m}
$$

As in the static case, $c^{d}(\sigma)$ is higher if the virus is more likely to generate severe health outcomes (hospitalization and/or death), if the morbidity discount is larger, if the public intervention is more effective, and if income is higher. In addition, some new comparative statics are of interest. For a very patient individual (i.e., with a low $r$ ) the possibility of dying sooner due to contracting the 


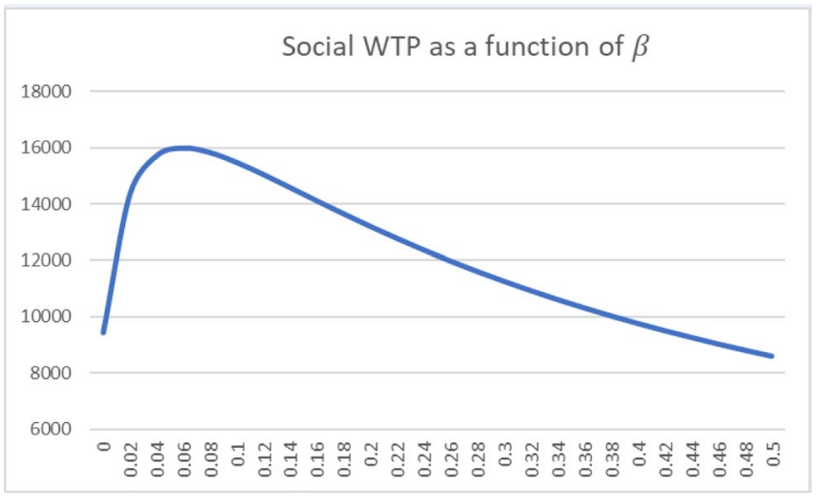

Fig. 2 Social WTP as a function of $\beta$

virus has a very large consequence in terms of the present value of the lost utility stream. However, as long as he recovers, being sick generates a small cost relative to the present value of his lifetime utility, especially if he is only avoiding being sick now rather than later (e.g., when vaccine discovery is unlikely to happen and the intervention is relatively ineffective). Conversely, a very impatient individual faces a relatively large cost of being sick (e.g., in the limit, as $r \rightarrow \infty$ and $m=0$, it is just like dying) but a relatively less significant consequence of dying. We conclude that the effect of greater impatience on the WTP $c(\sigma)$ is ambiguous (see Fig. 1 in Sect. 3).

Similarly, an increase in the probability of vaccine discovery generates a strong incentive to avoid being infected in the present to make use of the soonto-be-discovered vaccine. However, if there is a small likelihood of being infected over the course of the pandemic (i.e., at relatively high levels of $\beta$ ), the economic incentive to permanently reduce $p_{\mathrm{I}}$ decreases (e.g., when $\beta=1$ the only incentive to sacrifice income is to reduce the current risk of infection) (see Fig. 2 in Sect. 3).

Finally, in contrast to the static case, a higher baseline probability of infection also has an ambiguous effect on the WTP. As in the static case, a higher infection risk generates a stronger incentive to reduce it since being healthy is better than being sick (obviously, at $p_{\mathrm{I}}=0$ there is no incentive to pay anything). In addition, a higher infection risk generates a steeper infection curve. Given a relatively high probability of vaccine discovery (and/or health-care capacity shortages as below), flattening the epidemic curve is very valuable, so there is a dynamic effect which makes WTP to increase further as $p_{\mathrm{I}}$ increases. However, when flattening the curve is not very valuable (e.g., when $\beta$ and $r$ are relatively low and $K$ is large), the incentive to pay for an intervention that reduces $p_{\mathrm{I}}$ may be lower at higher levels of $p_{\text {I }}$ (see Fig. 3 in Sect. 3).

Remark Differences in WTP given different infection rates may explain the disjointed response to COVID-19 across U.S. States and, in particular, the greater acceptance of social distancing measures in States with a relatively large number of cases. As pointed out by an anonymous referee to us "the real source of the 


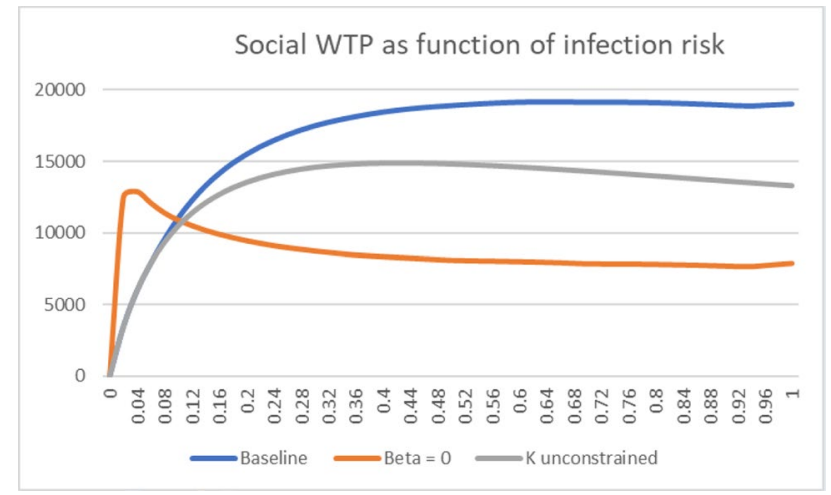

Fig. 3 Social WTP as a function of infection risk

discord may not be a tension between those people who are less vulnerable to the disease and those people who are more vulnerable to the disease but rather a tension between those states who are at the peak stage and those states who have not yet reached their peak stage, such that even if willingness to pay at the peak is the same, current WTP is not."

\subsubsection{The complete model}

The final specification of social welfare, which we use in the calibration, introduces heterogeneity and capacity constraints to the dynamic setting presented above. Social welfare can be written as follows:

$$
V^{S, F}\left\{p_{\mathrm{I}}\right\}=(M-N) w\left(\frac{1+r}{r}\right)+N\left[(1-\alpha) V^{l, F}\left\{p_{\mathrm{I}}\right\}+\alpha V^{h, F}\left\{p_{\mathrm{I}}\right\}\right],
$$

where $w\left(\frac{1+r}{r}\right), V^{l, F}\left\{p_{\mathrm{I}}\right\}$, and $V^{h, F}\left\{p_{\mathrm{I}}\right\}$ represent lifetime expected utility for the non-susceptible population, the low-risk population, and the high-risk population, respectively. The social WTP out of current income $c^{S, F}(\sigma)$ is

$$
V^{S, F}\left\{p_{\mathrm{I}}\right\}=V^{S, F}\left\{p_{\mathrm{I}}(1-\sigma), c^{S, F}(\sigma)\right\}
$$

In the Appendix, we present an explicit solution for the social WTP. As in the static case, $c^{S, F}(\sigma)$ increases with the size of the vulnerable population and when hospital capacity is more limited. In addition, since capacity shortages are more detrimental to high-risk individuals, who are more likely to need hospitalization and also more likely to die when the health-care system is overwhelmed, the effect of capacity constraints on $c^{S, F}(\sigma)$ is stronger when the proportion of vulnerable individuals is higher. Health-care capacity shortages also have a stronger effect on $c^{S, F}(\sigma)$ when people are more patient and/or when the probability of vaccine discovery is low since in those cases future capacity shortages are more relevant in the present (see Fig. 4 in Sect. 3).

Table 1 summarizes all the comparative statics that apply to $c^{S, F}(\sigma)$. 


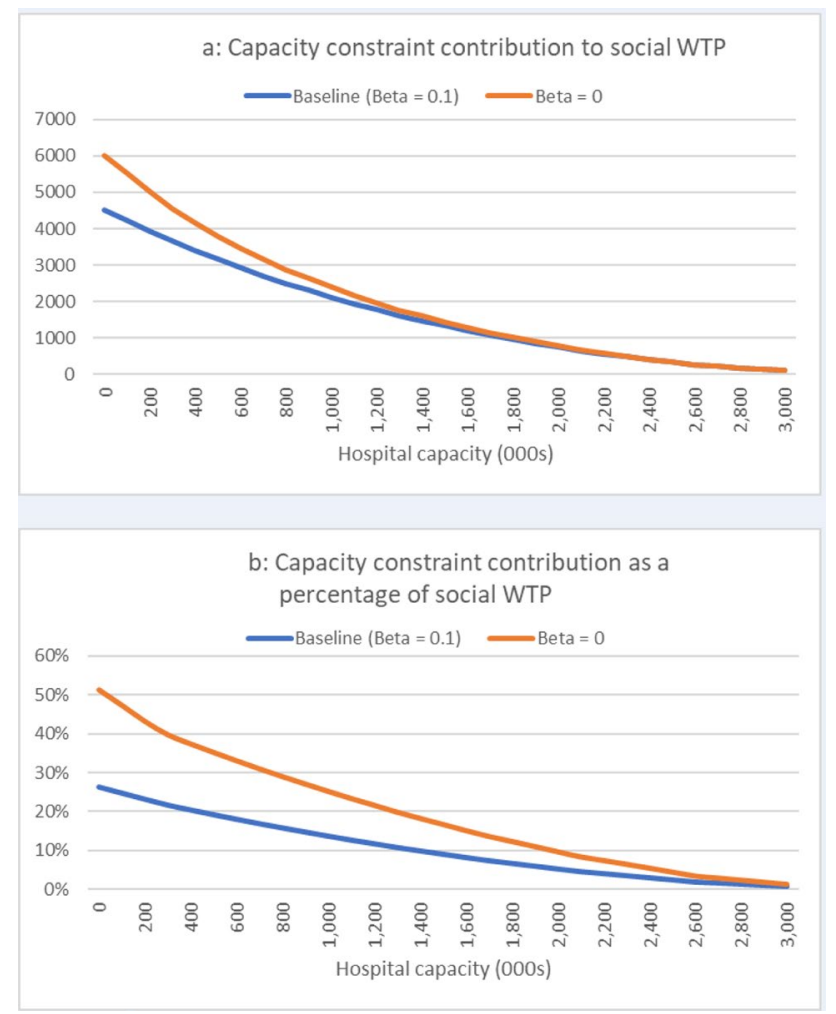

Fig. 4 a Capacity constraint contribution to social WTP. b Capacity constraint contribution as a percentage of social WTP

Table 1 Comparative statics analysis

\begin{tabular}{lll}
\hline Notation & Description & $\begin{array}{l}\text { Comparative } \\
\text { statics on } \\
\text { WTP }\end{array}$ \\
\hline $1-\sigma$ & Intervention factor & Negative \\
$N$ & Population initially susceptible & Positive \\
$w$ & Income (per period, per person) & Positive \\
$r$ & Period discount rate & Ambiguous \\
$m$ & Morbidity utility discount & Negative \\
$\beta$ & Probability of vaccine discovery & Ambiguous \\
$K$ & Hospital capacity & Negative \\
$p_{\mathrm{I}}$ & Baseline probability of infection & Ambiguous \\
$p_{\mathrm{S}}$ & Baseline probability of severe case & Positive \\
$p_{\mathrm{D}}$ & Baseline mortality rate of severe cases (given hospitalization) & Positive \\
$p_{\mathrm{DK}}$ & Baseline mortality rate of severe cases (given no hospitalization) & Positive \\
$\alpha$ & Proportion of vulnerable individuals among the initially susceptible & Positive \\
$\omega_{j}(j=S, D, D K)$ & Additional risks for type h & Positive \\
\hline
\end{tabular}


Table 2 Baseline calibration values

\begin{tabular}{|c|c|c|}
\hline Notation & Description & Baseline calibration values \\
\hline $1-\sigma$ & Intervention factor & 0.1 \\
\hline$M$ & Population & $325,000,000$ \\
\hline$N$ & Population initially susceptible & $0.8 M=261,600,000$ \\
\hline$w$ & Income (per period, per person) & $\$ 16,000$ \\
\hline$r$ & Period discount rate & 0.01 \\
\hline$m$ & Morbidity utility discount & 0 \\
\hline$\beta$ & Probability of vaccine discovery & 0.1 \\
\hline$K$ & Hospital capacity & $1,000,000$ \\
\hline$p_{\mathrm{I}}$ & Baseline probability of infection & 0.2 \\
\hline$p_{\mathrm{S}}$ & Baseline probability of severe case & 0.03 \\
\hline$p_{\mathrm{D}}$ & $\begin{array}{l}\text { Baseline mortality rate of severe cases (given hospitali- } \\
\text { zation) }\end{array}$ & 0.048 \\
\hline$p_{\mathrm{DK}}$ & $\begin{array}{l}\text { Baseline mortality rate of severe cases (given no hospi- } \\
\text { talization) }\end{array}$ & $1.5 p_{\mathrm{D}}$ \\
\hline$\alpha$ & $\begin{array}{l}\text { Proportion of vulnerable individuals among the initially } \\
\text { susceptible }\end{array}$ & 0.25 \\
\hline$\omega_{j}(j=S, D, D K)$ & Additional risks for type $h$ & $\begin{array}{l}\omega_{\mathrm{S}}=0.13 ; \\
\omega_{\mathrm{D}}=0.16 ; \\
\omega_{\mathrm{DK}}=1.5 \omega_{\mathrm{D}}=0.24\end{array}$ \\
\hline
\end{tabular}

Remark: In the calibration below, we present the social WTP per expected morbidity avoided, denoted $S_{\mathrm{VSI}}(\sigma)$ and defined as follows, $S_{\mathrm{VSI}}(\sigma)=c^{S, F}(\sigma) M / \Delta N_{\mathrm{I}}(\sigma)$, where $\Delta N_{\mathrm{I}}(\sigma)$ is the change in the expected number of people infected between the unmitigated scenario and the scenario with intervention parameter $\sigma$. This measure corresponds to what is known as the value of a statistical injury (e.g., Viscusi and Aldy (2003) and Viscusi and Gentry (2014)). Similarly, we calculate the WTP per expected morbidity avoided for each risk-type, $S_{\mathrm{VSI}}^{h}(\sigma)=c^{h, F}(\sigma) \alpha N / \Delta N_{I, h}(\sigma)$ and $S_{\mathrm{VSI}}^{l}(\sigma)=c^{l, F}(\sigma)(1-\alpha) N / \Delta N_{I, h}(\sigma)$.

\section{Calibration}

In this section, we calibrate the model to provide tentative estimates on the WTP to reduce morbidity and mortality risks during the pandemic COVID-19. We use the United States as a case study, although in the sensitivity analysis we evaluate a wide range of scenarios. The baseline calibration values are presented in Table 2. We take as the high-risk group those over 60 years of age, which comprises about $22 \%$ of the population, and an additional $3 \%$ of the population to account for other vulnerable individuals (e.g., young individuals with pre-existing conditions). ${ }^{7}$ The time period

\footnotetext{
${ }^{7}$ We do not consider differences by gender, although there seems to be evidence of significantly higher death rates for males [e.g., Jin et al (2020)].
} 
is taken to be 3 months (E.g., first period refers to February 2020 to April 2020). For the baseline epidemiological parameters, we use estimates in Verity et al. (2020). For the parameter $p_{\mathrm{S}}$, we use as a baseline 3\% for the low-risk group and $16 \%$ for the high-risk group, which is approximately the proportion of infected individuals hospitalized presented in Verity et al. (p. 7). We calculate $p_{\mathrm{D}}$, the fatality rate among those hospitalized, using the infection fatality rate in Verity et al. (Pg. 5) (0.145\% for the low-risk/under-60 group and 3.28\% for the high-risk/over-60 group) and dividing it by the hospitalization rate. We assume that a severe case that is not hospitalized has a $50 \%$ higher fatality rate, so $p_{\mathrm{DK}}=1.5 p_{\mathrm{D}}$.

We assume as a baseline that $80 \%$ of the population is susceptible when the intervention is considered, i.e., $N=0.8 M$. The rest of the population is composed of individuals that are never infected, individuals that have recovered and not infectious, and, most significantly for a novel virus like COVID-19 and in the initial stage of a pandemic, individuals that are infected and spread the virus before the intervention are in place. Based on this last group, we assume that the probability of infection in the initial period equals the ratio of those infected before the intervention to the total population; namely, $p_{\mathrm{I}}=0.2$.

Without taking into account capacity constraints, these parameters generate about 53 million infected in the initial period and 1.72 million deaths over the course of the epidemic in the unmitigated scenario, with about $88 \%$ deaths from the high-risk group. Assuming a capacity of 1 million hospital beds per period, another 450,000 deaths are predicted. These numbers are consistent with Ferguson et al.'s (2020) estimate of 2.2 million deaths in the unmitigated scenario.

With an intervention factor of 0.1 (i.e., a 90\% reduction in infection risk), the number of expected infected individuals in the first period decreases to 5.3 million and the number of deaths over the course of the pandemic decreases to about 172,000 (about 2 million lives saved), which seems to be somewhat consistent with current estimates. ${ }^{8}$

We set the probability of vaccine discovery $\beta$ at 0.1 , or about 2.5 years on expectation. For the preference parameters, we use $r=0.01$ (around $4 \%$ discount per year) and $m=0$ (i.e., zero utility during the single time period of the disease). Finally, we set $w=16,000$, about quarterly GDP per capita in the US in 2019.

This calibration generates a social WTP of $\$ 15,468$ per person, or about $\$ 5.0$ trillion (24\% of 2019 GDP), with $\$ 2,119$ arising as a result of expected capacity shortages. There is a large difference in the different groups' WTP, with a WTP of $\$ 41,024$ for the high-risk individuals, $\$ 10,868$ for the low-risk individuals, and (by construction) $\$ 0$ for those immune.

In terms of WTP per expected morbidity avoided, the calibration generates estimates of $S_{\mathrm{VSI}}(\sigma)=\$ 35,490, S_{\mathrm{VSI}}^{h}(\sigma)=\$ 84,975$, and $S_{\mathrm{VSI}}^{l}(\sigma)=\$ 19,188$. In addition, the calibration generates a social WTP per life saved of about $\$ 2.4$ million. As

\footnotetext{
8 As of May 2020, there were about 1,000,000 confirmed cases in the US. This would give a ratio of about 5 true cases per confirmed case, which is in the lower end of the estimates (e.g., Li et al (2020) estimate that in the early stages of the epidemic in China about $86 \%$ of the cases went undetected). As of mid-June 2020, there were 2 million confirmed cases and 120,000 confirmed deaths.
} 
Table 3 Lower WTP calibrations

\begin{tabular}{|c|c|c|c|c|}
\hline Description & Low WTP calibrations & Social WTP & High-risk WTP & Low-risk WTP \\
\hline Intervention factor & 0.25 & 12,691 & 38,777 & 8402 \\
\hline Period discount rate & 0.05 & 8262 & 15,487 & 8664 \\
\hline $\begin{array}{l}\text { Fraction of highly vulnerable } \\
\text { individuals }\end{array}$ & 0.1 & 10,857 & 43,354 & 10,323 \\
\hline $\begin{array}{l}\text { Probability of vaccine dis- } \\
\text { covery }\end{array}$ & 0.3 & 11,254 & 33,907 & 7531 \\
\hline Hospital capacity & Unconstrained & 13,517 & 37,646 & 10,071 \\
\hline $\begin{array}{l}\text { Baseline probability of infec- } \\
\text { tion }\end{array}$ & 0.1 & 10,994 & 31,443 & 7880 \\
\hline $\begin{array}{l}\text { Baseline probabilities } p_{j} \\
\quad(j=S, D, D K)\end{array}$ & 0.5 baseline $p_{j}$ & 14,282 & 44,140 & 9186 \\
\hline Additional risks for type $h$ & 0.5 baseline $\omega_{j}$ & 10,503 & 21,684 & 10,349 \\
\hline
\end{tabular}

Table 4 Higher WTP calibrations

\begin{tabular}{lllll}
\hline Description & High WTP calibrations & Social WTP & High-risk WTP & Low-risk WTP \\
\hline Intervention factor & 0.05 & 17,158 & 50,871 & 11,698 \\
$\begin{array}{l}\text { Period discount rate } \\
\text { Fraction of highly vulnerable }\end{array}$ & 0.005 & 24,064 & 84,267 & 12,181 \\
$\quad$ individuals & 0.5 & 23,756 & 49,056 & 10,576 \\
$\begin{array}{l}\text { Probability of vaccine dis- } \\
\quad \text { covery }\end{array}$ & 0.05 & 15,928 & 47,772 & 10,730 \\
$\quad \begin{array}{l}\text { Hospital capacity } \\
\quad \text { Baseline probability of infec- }\end{array}$ & 0.4 & 16,980 & 53,240 & 10,669 \\
$\quad$ tion & & 18,430 & 57,446 & 11,824 \\
$\quad \begin{array}{l}\text { Baseline probabilities } p_{j} \\
\quad(j=S, D, D K)\end{array}$ & 1.5 baseline $p_{j}$ & 17,094 & 47,794 & 12,675 \\
$\quad$ Additional risks for type $h$ & 1.5 baseline $\omega_{j}$ & 23,180 & 84,800 & 10,524 \\
\hline
\end{tabular}

discussed below in Sect. 4, these estimates are consistent with, although in the lower range, VSI and VSL estimates typically found in the literature.

Tables 3 and 4 present alternative calibrations. In Table 3, we consider parameter changes that generate lower WTP estimates while Table 4 presents parameter changes that generate higher WTP estimates. The social WTP ranges from about $\$ 8000$ in Table 3 to about $\$ 24,000$ in Table 4 (\$2.7 T to $\$ 7.8 \mathrm{~T})$. For the high-risk group the range is significantly larger, from about $\$ 15,500$ to $\$ 84,500$ (and only about $\$ 7,500$ to $\$ 12,500$ for the low-risk group).

Given the calibration selected, willingness to pay is most sensitive to changes in the discount rate, the proportion-vulnerable individuals, and their risk of hospitalization and death, although changes in the latter generate post-intervention attack rates and deaths that are significantly below or above current estimates. The probability 
of vaccine discovery and hospital capacity also have a significant effect on WTP, although the effects are less pronounced.

Figures 1, 2 and 3 further inspect how WTP varies as a few key parameters change. Figure 1a shows that a higher discount rate reduces the social WTP monotonically given the numbers in the baseline calibration in Table 2. Figure 1b shows, however, that given a low probability of vaccine discovery and relatively ineffective interventions, a higher discount rate may increase the social WTP. Figure 2 confirms the intuition that a higher probability of vaccine discovery increases the incentive to push the risk forward but at the same time reduces the overall risk of future infections (i.e., there are fewer lives to be saved), thereby reducing the social WTP at sufficiently high levels of $\beta$. Figure 3 shows the ambiguous impact of the baseline probability of infection on the social WTP. As discussed in Sect. 2.2., given a strong incentive to flatten the pandemic curve, social WTP tends to increase with a higher probability of infection. However, when that incentive is reduced, as is the case when vaccine discovery is unlikely or capacity constraints are less important, a higher infection risk may result in a lower social WTP.

Figures $4 \mathrm{a}$ and $4 \mathrm{~b}$ show the contribution of expected health-care capacity shortages to social WTP (i.e., how much lower WTP would be if capacity was unconstrained) and the contribution as a percentage of social WTP, respectively, for different levels of available capacity and for two different probabilities of vaccine discovery. Noticeable, as described in Sect. 2.2.2, capacity constraints play a much bigger role when vaccine discovery is unlikely. For instance, for $K=100,000$ the capacity constraint contributes about $25 \%$ of the social WTP if $\beta=0.1$ but more than $50 \%$ when $\beta=0$, for the baseline value $K=1,000,000$ the capacity constraint contributes about $15 \%$ of the social WTP if $\beta=0.1$ but more than $25 \%$ when $\beta=0$.

\section{Discussion and conclusions}

Key characteristics of pandemics such as COVID-19 include significant uncertainty over the timing of vaccine discovery, the possibility of health-care capacity shortages, and large heterogeneity in the degree of vulnerability to virus infection. In this paper, we presented a model that incorporates these features to the standard WTP model of morbidity/fatality-risk reduction and we presented some suggestive evidence of the significance of these features.

We showed that the social WTP for a sizeable reduction in infection risk during a pandemic can be quite large, in the range of $\$ 3 \mathrm{~T}$ to $\$ 7 \mathrm{~T}$ (15 to 35\% of 2019 U.S. GDP). The social WTP is particularly large at low levels of the social discount rate, while the probability of vaccine discovery and the risk of health-care capacity shortages also play a significant role. Are the potential benefits large enough to justify the measures adopted? On top of revealed preference, the answer seems to be positive. For example, Barro et al. (2020a, b) argue that the costs of the Great Influenza Pandemic of 1918-1920 represent a plausible worst-case scenario for a large epidemic like COVID. In that case, they estimate that GDP decreased by about $6 \%$ and consumption by about $8 \%$. In the case of COVID, first-quarter GDP in the U.S. contracted by almost $5 \%$ and consumption by almost $8 \%$, and there are arguably 
many other costs beyond reduced economic activity, but to the extent that economic restrictions are not extended for a long period, all measures indicate that the costs will be below the expected benefits in terms of lives saved and disease costs avoided -at least on an economic/statistical sense.

While the estimated benefits of mitigation are large in absolute terms, and in line with other papers that have performed cost-benefit analyses in the context of COVID-19 [e.g., Greenstone and Nigam (2020), Scherbina (2020) and Thunström et al. (2020)], they are somewhat lower than typical estimates of the value of statistical injury found in the literature when considering the large number of infections avoided. For example, compared to our estimates $\left(S_{\mathrm{VSI}}(\sigma)=\$ 35,490\right.$, $S_{\mathrm{VSI}}^{h}(\sigma)=\$ 84,975$, and $\left.S_{\mathrm{VSI}}^{l}(\sigma)=\$ 19,188\right)$, Viscusi and Aldy (2003) find that most studies have estimates of VSI in the range of $\$ 29,000-\$ 100,000$ per injury (in 2020 dollars). The U.S. Department of Health and Human Services (2016) recommends valuing a quality-adjusted-life-year at approximately $\$ 250,000-\$ 800,000$ (2020 dollars), which, given our assumption of zero utility during the three months in which the individual gets sick, would correspond to a loss of $\$ 62,500-\$ 200,000$ for those infected. As another reference point, out calibration generates a social WTP per life saved of about $\$ 2.4$ million, which is also in the lower range of current VSL estimates [e.g., Viscusi and Aldy (2003), Robinson and Hammitt (2016) and U.S. Department of Health and Human Services (2016)].

The likely underestimation of VSI and VSL could be the result of our simplifying assumptions on individual and social preferences. In particular, a higher WTP than the one we estimated may be justified if individuals are risk averse, if there is uncertainty over the epidemiological parameters and individuals are ambiguity averse [see Treich (2010) and Bleichrodt et al. (2019)]—-something which is likely to play an important role at the beginning of the pandemic —or if the social utility function assigns greater weight to those more vulnerable.

Another key simplifying assumption of our model is that the infection rate remains constant once an intervention is in place. ${ }^{9}$ This assumption allowed us to derive closed form solutions and to provide insights of the comparative statics of vaccine discovery, health-care capacity constraints, and others. However, our model may miss some of the significant insights provided by economic-epidemiological models which place greater emphasis on the short-term dynamics of the epidemic [e.g., to evaluate the optimal mitigation policy over the course of the pandemic, as done, for example, by Acemoglu et al. (2020), Alvarez et al. (2020), and Jones et al. (2020)].

Finally, a key assumption driving large differences in WTP is that WTP differs by the degree of disease vulnerability but not (inversely) by age. If willingness to pay is lower at an old age, as some studies suggest it is [see e.g., Aldy and Viscusi (2008)], our calibration may overestimate differences in WTP and also the social WTP (e.g., the elderly, who are largely vulnerable, may be willing to pay as much as the young, who are largely relatively safe). In effect, since the cost of social distancing is likely

\footnotetext{
${ }^{9}$ In standard epidemiological models (e.g., the SIR model) the likelihood of infection depends on the stocks of infected and susceptible individuals at any given time (see Atkeson (2020) for an introduction to SIR models).
} 
age-dependent, a more effective alternative to the uniform policies studied here and adopted by many countries would seem to be the adoption of an age-dependent mitigation strategy, with stricter confinement of senior citizens [see e.g., Acemoglu et al. (2020) and Gollier (2020)]. Or, to put it differently, the use of strong, uniform policies may impose an unnecessarily large redistribution of well-being from young, healthy individuals to groups of vulnerable individuals.

In sum, our analysis is suggestive, but preliminary. Much theoretical and empirical analysis remains to be done to evaluate the proper policies to reduce morbidity and mortality risks during an epidemic and, at least as importantly, to analyze the pecuniary and non-pecuniary costs of those interventions.

\section{Compliance with ethical standards}

Conflict of interest The authors declare that they have no conflict of interest.

\section{Appendix: derivations of the main equations in the text}

\section{Equation 4}

Given a probability of hospitalization $p_{h}=\min \left(1, \frac{K}{p_{\mathrm{S}} p_{\mathrm{I}} M}\right)$, expected utility can be written as

$$
V^{K}\left\{p_{\mathrm{I}}\right\}=\left(1-p_{\mathrm{I}} p_{\mathrm{S}}\right) W+p_{\mathrm{I}} p_{\mathrm{S}} m W\left(p_{h}\left(1-p_{\mathrm{D}}\right)+\left(1-p_{h}\right)\left(1-p_{\mathrm{DK}}\right)\right) .
$$

Equivalently,

$$
V^{K}\left\{p_{\mathrm{I}}\right\}=\left(1-p_{\mathrm{I}} p_{\mathrm{S}}\right) W+p_{\mathrm{I}} p_{\mathrm{S}} m W\left(1-p_{\mathrm{D}}\right)+p_{\mathrm{I}} p_{\mathrm{S}} m W\left(p_{\mathrm{DK}}-p_{\mathrm{D}}\right)\left(1-p_{h}\right)
$$

If the capacity constraint does not bind, we have $p_{h}=1$ and

$$
V^{K}\left\{p_{\mathrm{I}}\right\}=\left(1-p_{\mathrm{I}} p_{\mathrm{S}}\right) W+p_{\mathrm{I}} p_{\mathrm{S}} m W\left(1-p_{\mathrm{D}}\right) .
$$

If the constraint binds, using the definition $G=p_{\mathrm{S}} p_{\mathrm{I}} M-K$ we obtain

$$
V^{K}\left\{p_{\mathrm{I}}\right\}=\left(1-p_{\mathrm{I}} p_{\mathrm{S}}\right) W+p_{\mathrm{I}} p_{\mathrm{S}} m W\left(1-p_{\mathrm{D}}\right)+m W\left(p_{\mathrm{DK}}-p_{\mathrm{D}}\right)\left(\frac{G}{M}\right),
$$

which is Eq. 4 in the text.

\section{Equation 5}

Willingness to pay $C^{K}(\sigma)$ can be written as follows

$$
\begin{aligned}
V^{b} & \left\{p_{\mathrm{I}}\right\}-m\left(p_{\mathrm{DK}}-p_{\mathrm{D}}\right) W \max (G, 0) / M \\
& =V^{b}\left\{p_{\mathrm{I}}(1-\sigma)\right\}\left(1-\frac{C^{K}(\sigma)}{W}\right)-m\left(p_{\mathrm{DK}}-p_{\mathrm{D}}\right)\left(W-C^{K}(\sigma)\right) \max \left(G^{\prime}, 0\right) / M
\end{aligned}
$$


Solving for $C^{K}(\sigma)$,

$$
C^{K}(\sigma)=\frac{V^{K}\left\{p_{\mathrm{I}}(1-\sigma)\right\}-V^{K}\left\{p_{\mathrm{I}}\right\}}{V^{K}\left\{p_{\mathrm{I}}(1-\sigma)\right\}} W
$$

\section{Equation 7}

Willingness to pay $C^{S}(\sigma)$ can be written as follows

$$
\begin{aligned}
(M-N) W+N\left[(1-\alpha) V^{b}\left\{p_{\mathrm{I}}\right\}+\alpha V^{h}\left\{p_{\mathrm{I}}\right\}\right] \\
\quad=\left(1-\frac{C^{S}(\sigma)}{W}\right)\left[(M-N) W+N\left[(1-\alpha) V^{b}\left\{p_{\mathrm{I}}(1-\sigma)\right\}+\alpha V^{h}\left\{p_{\mathrm{I}}(1-\sigma)\right\}\right]\right] .
\end{aligned}
$$

Solving for $C^{S}(\sigma)$

$$
\frac{N\left[(1-\alpha)\left[V^{b}\left\{p_{\mathrm{I}}(1-\sigma)\right\}-V^{b}\left\{p_{\mathrm{I}}\right\}\right]+\alpha\left[V^{h}\left\{p_{\mathrm{I}}(1-\sigma)\right\}-V^{h}\left\{p_{\mathrm{I}}\right\}\right]\right]}{\left[(M-N) W+N\left[(1-\alpha) V^{b}\left\{p_{\mathrm{I}}(1-\sigma)\right\}+\alpha V^{h}\left\{p_{\mathrm{I}}(1-\sigma)\right\}\right]\right]}=\frac{C^{S}(\sigma)}{W} .
$$

Rearranging,

$$
C^{S}(\sigma)=q_{l} \frac{\left[V^{b}\left\{p_{\mathrm{I}}(1-\sigma)\right\}-V^{b}\left\{p_{\mathrm{I}}\right\}\right]}{V^{b}\left\{p_{\mathrm{I}}(1-\sigma)\right\}} W+q_{h} \frac{\left[V^{h}\left\{p_{\mathrm{I}}(1-\sigma)\right\}-V^{h}\left\{p_{\mathrm{I}}\right\}\right]}{V^{h}\left\{p_{\mathrm{I}}(1-\sigma)\right\}} W
$$

with

$$
q_{h}=\frac{\alpha N V^{h}\left\{p_{\mathrm{I}}(1-\sigma)\right\}}{(M-N) W+N\left[(1-\alpha) V^{b}\left\{p_{\mathrm{I}}(1-\sigma)\right\}+\alpha V^{h}\left\{p_{\mathrm{I}}(1-\sigma)\right\}\right]}
$$

$q_{l}=\frac{(1-\alpha) N V^{b}\left\{p_{\mathrm{I}}(1-\sigma)\right\}}{(M-N) W+N\left[(1-\alpha) V^{b}\left\{p_{\mathrm{I}}(1-\sigma)\right\}+\alpha V^{h}\left\{p_{\mathrm{I}}(1-\sigma)\right\}\right]}$.

\section{Social WTP in the complete model (Eqs. 10 and 11)}

Recall that new cases at time $t$ equal $p_{\mathrm{I}}\left(1-p_{\mathrm{I}}\right)^{t} N$, of which a proportion $\left[(1-\alpha) p_{\mathrm{S}}+\alpha\left(p_{\mathrm{S}}+\omega_{\mathrm{S}}\right)\right]$ is hospitalized. Given period capacity constraints $K$, the probability of hospitalization at time $t$ is $p_{h, t}=\min \left(1, \frac{K}{p_{\mathrm{I}}\left(1-p_{\mathrm{I}}\right)^{t-1} N\left(p_{\mathrm{S}}+\alpha \omega_{\mathrm{S}}\right)}\right)$ and the capacity gap is $G_{t}=p_{\mathrm{I}}\left(1-p_{\mathrm{I}}\right)^{t-1} N\left(p_{\mathrm{S}}+\alpha \omega_{\mathrm{S}}\right)-K$. It follows that expected lifetime utility for type $l$ individuals $V^{l, F}\left\{p_{\mathrm{I}}\right\}$ can be written as follows:

$$
\begin{aligned}
V^{l, F}\left\{p_{\mathrm{I}}\right\}= & \frac{(1+r)}{r+p_{\mathrm{I}}+\beta\left(1-p_{\mathrm{I}}\right)} w\left[\left(1-p_{\mathrm{I}}\right)\left(1+\beta \frac{1}{r}\right)+p_{\mathrm{I}}\left(1-p_{\mathrm{S}} p_{\mathrm{D}}\right)\left(m+\frac{1}{r}\right)\right] \\
& -w\left(m+\frac{1}{r}\right) p_{\mathrm{I}} p_{\mathrm{S}}\left(p_{\mathrm{DK}}-p_{\mathrm{D}}\right) \sum_{t=1}^{\infty} \frac{(1-\beta)^{t-1}(1-\beta)^{t-1}}{(1+r)^{t-1}}\left(1-p_{h, t}\right),
\end{aligned}
$$

or equivalently, 


$$
\begin{aligned}
V^{l, F}\left\{p_{\mathrm{I}}\right\}= & \frac{(1+r)}{r+p_{\mathrm{I}}+\beta\left(1-p_{\mathrm{I}}\right)} w\left[\left(1-p_{\mathrm{I}}\right)\left(1+\beta \frac{1}{r}\right)+p_{\mathrm{I}}\left(1-p_{\mathrm{S}} p_{\mathrm{D}}\right)\left(m+\frac{1}{r}\right)\right] \\
& -w\left(m+\frac{1}{r}\right) p_{\mathrm{S}}\left(p_{\mathrm{DK}}-p_{\mathrm{D}}\right) \sum_{t=1}^{\infty} \frac{(1-\beta)^{t-1}}{(1+r)^{t-1}} \frac{\max \left(G_{t}, 0\right)}{N\left(p_{\mathrm{S}}+\alpha \omega_{\mathrm{S}}\right)} .
\end{aligned}
$$

Similarly, expected utility for high-risk individuals (i.e., those with probability of severe sickness $p_{\mathrm{S}}+\omega_{\mathrm{S}}$, mortality risk $p_{\mathrm{D}}+\omega_{\mathrm{D}}$ if hospitalized, and $p_{\mathrm{DK}}+\omega_{\mathrm{DK}}$ if not hospitalized) is

$$
\begin{aligned}
V^{l, F}\left\{p_{\mathrm{I}}\right\}= & \frac{(1+r)}{r+p_{\mathrm{I}}+\beta\left(1-p_{\mathrm{I}}\right)} w\left[\left(1-p_{\mathrm{I}}\right)\left(1+\beta \frac{1}{r}\right)+p_{\mathrm{I}}\left(1-\left(p_{\mathrm{S}}+\omega_{\mathrm{S}}\right)\left(p_{\mathrm{D}}+\omega_{\mathrm{D}}\right)\right)\left(m+\frac{1}{r}\right)\right] \\
& -w\left(m+\frac{1}{r}\right)\left(p_{\mathrm{S}}+\omega_{\mathrm{S}}\right)\left(\left(p_{\mathrm{DK}}+\omega_{\mathrm{DK}}\right)-\left(p_{\mathrm{D}}+\omega_{\mathrm{D}}\right)\right) \sum_{t=1}^{\infty} \frac{(1-\beta)^{t-1}}{(1+r)^{t-1}} \frac{\max \left(G_{t}, 0\right)}{N\left(p_{\mathrm{S}}+\alpha \omega_{\mathrm{S}}\right)} .
\end{aligned}
$$

Notice that, as stated in Sect. 2.2.2, capacity shortages (captured by the second term in the equations above) are more detrimental to high-risk individuals since they are more likely to need hospitalization (i.e., $\omega_{\mathrm{S}}>0$ ) and more likely to die (i.e., $\omega_{\mathrm{DK}}-\omega_{\mathrm{D}}>0$ ) when the health-care system is overwhelmed.

To simplify notation, let us suppose, as we do in the calibration, that $m=0$. It follows that social WTP out of current income is

$$
c^{S, F}(\sigma)=\frac{N(1-\alpha)\left[V^{l, F}\left\{p_{\mathrm{I}}(1-\sigma)\right\}-V^{l, F}\left\{p_{\mathrm{I}}\right\}\right]+N \alpha\left[V^{h, F}\left\{p_{\mathrm{I}}(1-\sigma)\right\}-V^{h, F}\left\{p_{\mathrm{I}}\right\}\right]}{(M-N)+N\left(1-p_{\mathrm{I}}(1-\sigma)\right)} .
$$

\section{References}

Acemoglu, D., V. Chernozhukov, I. Werning, and M.D. Whinston. 2020. A multi-risk SIR model with optimally targeted lockdown. NBER Working Paper No. 27102

Adler, M.D., J.K.M. Hammitt, and N. Treich. 2014. The social value of mortality risk reduction: VSL versus the social welfare function approach. Journal of Health Economics 35: 82-93.

Aldy, J.E., and W.K. Viscusi. 2008. Adjusting the value of a statistical life for age and cohort effects. Review of Economics and Statistics 90: 573-581.

Alvarez, F.E., D. Argente, and F. Lippi. 2020. A simple planning problem for Covid-19 lockdown. NBER Working Paper No. 26981

Anderson, R.M., H. Heesterbeek, D. Klinkenberg, and T.D. Hollingsworth. 2020. How will country-based mitigation measures influence the course of the COVID-19 epidemic? Lancet 395: 931-934.

Anderson, H., and N. Treich. 2011. The value of a statistical life. In Handbook in transport economics, ed. A. de Palma, R. Lindsey, E. Quinet, and R. Vickerman, 396-424. Cheltenham: Edward Elgar.

Atkeson, A. 2020. What will be the economic impact of COVID-19 in the US? Rough estimates of disease scenarios. NBER Working Paper No. 26867.

Barro, R.J, J.F Ursúa, and J. Weng. 2020a. The coronavirus and the great influenza pandemic. Lessons from the "spanish flu" for the coronavirus's potential effects on mortality and economic activity. NBER working paper 26866

Barro, R.J, J.F Ursúa, and J. Weng. 2020b. Coronavirus meets the great influenza pandemic. VOX CEPR Policy Portal. March 2020. https://voxeu.org/article/coronavirus-meets-great-influenza-pandemic 
Bleichrodt, H., C. Courbage, and B. Rey. 2019. The value of a statistical life under changes in ambiguity. Journal of Risk and Uncertainty 58: 1-15.

Ferguson, N.M., D. Laydon, G. Nedjati-Gilani, N. Imai, K. Ainslie, M. Baguelin, S. Bhatia, A. Boonyasiri, Z. Cucunubá, G. Cuomo-Dannenburg, and A. Dighe. 2020. Impact of non-pharmaceutical interventions (NPIs) to reduce COVID-19 mortality and healthcare demand. Imperial College COVID-19 Response Team, London, March, 16. https://www.imperial.ac.uk/media/imperialcollege/medicine/sph/ide/gida-fellowships/Imperial-College-COVID19-NPI-modelling-16-032020.pdf.

Gollier, C. 2020. Age-specific deconfinement strategies. Covid Economics 24 (1-31): 2020.

Greenstone, M., and V. Nigam. 2020. Does social distancing matter? Becker Friedman Institute Working Paper 2020-26.

Jin, J.-M., P. Bai, W. He, et al. 2020. Gender differences in patients with COVID-19: Focus on severity and mortality. Frontiers in Public Health. https://doi.org/10.3389/fpubh.2020.00152.

Jones, C.J., T. Philippon, and V. Venkateswaran. 2020. Optimal mitigation policies in a pandemic: Social distancing and working from home. NBER Working Paper No. 26984

Li, R., S. Pei, B. Chen, Y. Song, T. Zhang, W. Yang, and J. Shaman. 2020. Substantial undocumented infection facilitates the rapid dissemination of novel coronavirus (SARS-CoV2). Science. https:// doi.org/10.1126/science.abb3221.

Robinson, L.A., and J.K. Hammitt. 2016. Valuing reduction in fatal illness risks: Implications of recent research. Health Economics 25: 1039-1052.

Scherbina, A. 2020. Determining the optimal duration of the Covid-19 suppression policy: a cost-benefit analysis

Shepard, D., and R. Zechhauser. 1984. Survival versus consumption. Management Science 30: 423-439.

Thunström, L., S.C. Newbold, D. Finnoff, M. Ashworth, and J.F. Shogren. 2020. The benefits and costs of using social distancing to flatten the curve for Covid-19. Journal of Benefit Cost Analysis pp. $1-27$

Treich, N. 2010. The value of a statistical life under ambiguity aversion. Journal of Environmental Economics and Management 59 (1): 15-26.

Treich N., and Y. Yang. 2019. Public safety and imperfect taxation. Working paper. https://yutingyang .com/wp-content/uploads/2020/04/Research_Public-safety-under-imperfect-taxation-_NTYY.pdf

U.S. Department of Health and Human Services. 2016. Guidelines for regulatory impact analysis. https:// aspe.hhs.gov/pdf-report/guidelines-regulatory-impact-analysis

Verity, R., L.C. Okell, I. Dorigatti, et al. 2020. Estimates of the severity of coronavirus disease 2019: a model-based analysis, 1473-3099. ISSN: Lancet Infectious Diseases.

Viscusi, W.K. 2014. The value of individual and societal risks to life and health. In Handbook of the economics of risk and uncertainty, chapter 7, ed. M. Machina and K. Vicusi. Amsterdam: North-Holland.

Viscusi, W.K., and J.E. Aldy. 2003. The value of a statistical life: A critical review of markets estimates throughout the world. Journal of Risk and Uncertainty 27: 5-76. 